\title{
Climate Science Needs to Take Risk Assessment Much More Seriously
}

\author{
ROWAN T. SUTTON
}

C limate science has achieved a great deal. It has demonstrated unequivocally that human activities have been the major driver of climate change since the mid-twentieth century (IPCC 2013), and there has been considerable-if insufficient-progress toward global actions to address the problems arising. But following the Paris Agreement, what are the priorities for climate research? Which concerns should guide the further development of global climate models? And what are the consequences for climate assessments, especially those of the Intergovernmental Panel on Climate Change (IPCC)?

From the perspective of societal needs-that is, the needs of decision-makers in governments, businesses, or civil society-climate change is a problem in risk assessment ${ }^{1}$ and risk management. Therefore, a central question is, What information can science provide to meet these needs? In this article, I want to focus particularly on the contribution of physical climate science, and the community of scientists represented by IPCC Working Group I (WGI). It is notable that the requirements to inform risk assessment have had little prominence in the WGI contributions to the major IPCC assessment reports, such as the most recent fifth report (IPCC 2013). By contrast, these requirements were highlighted prominently in the Summary for Policymakers of the Working Group II (WGII) contribution (IPCC 2014). This article explores the reasons why physical climate science has not paid more attention to risk assessment and argues that this situation should be remedied urgently. It also discusses some of the implications for priorities in climate research and modeling, and for IPCC climate assessments.

PRINCIPLES OF RISK ASSESSMENT. In simple terms, a risk is "something bad that might happen"

\footnotetext{
${ }^{1}$ Assessment of opportunities is also important and can be considered within a similar framework, but is not a focus of this article.
}

(King et al. 2015). Risk assessment requires information about 1) what events are possible, 2) how likely they are, and 3) what the impacts or consequences could be. A common measure of the risk associated with a specific event is risk = likelihood $\times$ impact, which highlights the importance of considering likelihood and impact together. Risk assessment cannot be done properly by focusing on only one of these factors or by considering them only sequentially: for risk, the interaction between them matters.

A landmark climate change risk assessment was published by King et al. (2015). The introduction to this report summarizes key principles of risk assessment as follows:

1) Identify risks in relation to objectives (e.g., protect human prosperity and security).

2) Identify the biggest risks.

3) Consider the full range of probabilities.

4) Use the best available information.

5) Take a holistic view (i.e., consider all relevant factors).

6) Be explicit about value judgements.

Risk assessment is invariably a multidisciplinary task: the necessary information can only be obtained by drawing together the expertise from more than one community. For example, physical climate science can provide information about future climate, while

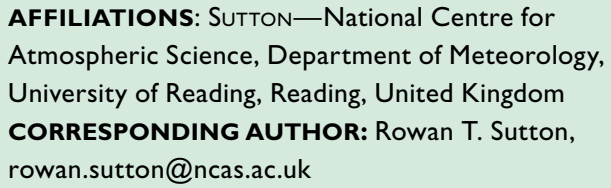


biological, economic, and social sciences are required to assess the full range of impacts and consequences. However, there is no single "best" measure of impact: as is highlighted by principles 1 and 6 , impact is ultimately a consequence of choices about values and objectives.

A key consequence of principles 2 and 3 is the importance of paying specific attention to high-impact events, even if their likelihood is considered low. Insuring homes against fire risk is a standard example: most people buy such insurance not because they consider it likely that their house will burn down but rather because their potential loss is very great.

Concerning principles 3 and 4, quantitative information-where it is available-is always desirable. However, another important insight from the literature on risk assessment is that qualitative information can still be very valuable. In particular, there are many situations in which only qualitative assessments of likelihood are possible (e.g., Weaver et al. 2013, 2017; Shepherd et al. 2018). In such situations, it is common to use qualitative tools, such as discrete scenarios. A scenario describes a plausible sequence of future events but is not associated with a specific probability. However, the impacts arising from different scenarios can be explored in detail. If scenarios are designed well, they are very useful to inform decision-making.

\section{WHY HAS PHYSICAL CLIMATE SCIENCE NOT PAID MORE ATTENTION TO RISK ASSESS-}

MENT? Why did the IPCC not long ago produce a risk assessment like that of King et al. (2015)? A key reason is the "siloing" of expertise between the three working groups, which has inhibited the necessary integration of knowledge from different disciplines (i.e., taking a "holistic view"). This siloing has been exacerbated by a scoping process for the major assessment cycles that remains too "bottom-up," starting with the scientists rather than with the needs of decision-makers. One peculiarity of this process is that scoping of the headline synthesis report occurs only long after the scoping of the individual working group reports. To meet the needs of decision-makers more effectively, it should be the other way around. It is important to acknowledge that the recent crosscutting reports (e.g., IPCC 2012, 2018) are evidence of significant progress in the IPCC addressing the needs for risk assessment more effectively, but the cycle of major assessment reports is continuing in the Sixth Assessment without substantial changes to the process.
One consequence of the siloing of climate science is that WGI scientists have tended to assume that risk assessment is not their business because it requires information about impacts that is possessed by WGII. However, this is incorrect. Impacts and risks can-indeed must-be assessed, and where possible quantified, using a wide range of metrics. WGI is the appropriate community to assess risks in terms of climate variables (e.g., temperatures, carbon budgets, extreme weather), especially variables that are relevant to a wide range of decisions in connection with adaptation or mitigation.

An additional-related-reason that WGI has paid little attention to risk assessment is the widespread view that the primary job of the WGI community is to provide predictions and projections (i.e., predictions conditioned on socioeconomic scenarios) rather than risk assessments. ${ }^{2}$ The WGI community has focused large resources on attempts to "quantify the uncertainty in climate predictions/projections" (e.g., Hawkins and Sutton 2009), that is, on quantifying likelihoods, with little attention to impacts. This focus reflects the strong influence of meteorology on the development of climate science. Predictions in meteorology involve using models to propagate forward information about the current state of the atmosphere (i.e., initial conditions) to generate quantitative estimates of its state and uncertainty at a future time, often expressed in terms of quantified likelihoods. Confidence that these likelihoods are meaningful relies either on 1) repeating the prediction process sufficiently often that skill and reliability can be demonstrated robustly or 2) arguments and evidence that the relevant uncertainties can all be quantified, at least in principle. Unfortunately, neither of these conditions holds for statements about future climate change, at least for lead times beyond a decade or so. Anthropogenic climate change is a unique experiment, and there is a significant body of research demonstrating that there are no adequate methods to quantify all the epistemic uncertainties associated with the climate response to anthropogenic greenhouse gas forcing (e.g., those related to processes missing from all climate models), even at a global scale; for regional and smaller scales, the problem is much worse.

The impossibility of quantifying precisely the likelihood that future climate change will have a

\footnotetext{
2 See, for example, the WCRP Strategic Framework 2005-15: Coordinated Observation and Prediction of the Earth System.
} 
particular magnitude (or other specific features) does not, of course, mean we have no information about it. Scientific arguments and evidence can often provide bounds or-in IPCC terminology-a "likely range" for key parameters such as global mean temperature. And, of course, if we can acquire new evidence that enables narrowing such bounds, this is progress. However, we should not imagine that it will ever be possible to provide detailed and meaningful probability distribution functions (PDFs) for future climate change analogous to those that-at least in principle-are possible for short-range weather forecasts.

A final reason that may have contributed to the WGI community neglecting the needs of risk assessment is concern about accusations of scaremongering (e.g., Sutton 2018). Risk assessment does involve drawing attention to potential "bad" outcomes even when they are very uncertain. Such an approach does not come naturally to many scientists who-for good reasons-are cautious by nature. The politicized debates around climate change have exacerbated this situation.

SOME CONSEQUENCES. The consequence of physical climate science paying little attention to the needs of risk assessment has been that important issues have been neglected. Two examples can illustrate this point.

One consequence has been to afford insufficient attention to the low-likelihood, high-impact events that-as already discussed-are a central concern in risk assessment. King et al. (2015) point out that decision-makers facing risks are typically most concerned with two questions: 1) What is likely? 2) How bad could it be/what must we avoid? The latter question is fundamental to the development of robust strategies for both adaption (e.g., resilience) and mitigation. However, WGI has focused overwhelmingly on question 1 (e.g., assessing the likely range for key parameters). But physical climate science has much knowledge and expertise to bring to question 2. It is essential that climate science identifies what is possible in the climate system, not merely what is likely (e.g., Weaver et al. 2013; Schellnhuber 2018). Possibilitieswhich come with the potential for surprises-are a major concern for risk assessment. Furthermore, there are no fundamental obstacles to including assessments of the relevant risks within WGI reports (Sutton 2018).

WGI has given some attention to the potential for "abrupt" climate change. However, abrupt changes are only a subset of low-likelihood, high-impact events and not necessarily the most important subset
(Sutton 2018). High climate sensitivity is an example of a very high-impact possibility that is not associated with any abrupt change in the Earth system. Furthermore, even when WG1 has considered low-likelihood, high-impact scenarios, it has tended to focus too narrowly on likelihood and given insufficient attention to impacts. Here is an example from the Fifth Assessment Report (AR5) Summary for Policymakers (SPM; IPCC 2013): "It is very unlikely that the AMOC will undergo an abrupt transition or collapse in the twenty-first century for the scenarios considered." No information whatsoever about the impacts of an Atlantic meridional overturning circulation (AMOC) collapse is communicated, despite the importance of impact information for decision-making. WGII is not the appropriate community to provide information about the magnitude of regional climate change or sea level rise that could result from a collapse of the AMOC, were it to occur; this responsibility sits squarely with the WGI, but WGI-both as a research community and in the production of IPCC reportshas not considered it a priority. This neglect must be remedied.

A second example is that physical climate science has until recently afforded surprisingly little attention to what is a key issue for many decision-makers, namely, quantifying current risks-that is, What is the current likelihood of specific high impact events? Such events are by definition rare; that is, they are associated with low likelihood. But whether this likelihood is 1 in 20, 1 in 200, or 1 in 200,000 is of great importance for those concerned with contingency planning and building resilience. In this case, the quantification of likelihoods is very important and is more tractable than for statements about future climate change. In a changing (nonstationary) climate, the appropriate likelihoods cannot be reliably estimated from historical data alone. A model of how climate change is affecting likelihoods (and risk) is required. For simple events (e.g., daily extremes of temperature), statistical models may suffice, but for more complex events (e.g., multivariate or correlated hazards), large ensembles of simulations with general circulation models are needed (Stott et al. 2016; Mizuta et al. 2017). The recent-climate component of the Japanese Database for Policy Decision-Making for Future Climate Change (d4PDF) program (Mizuta et al. 2017) is a pioneering example of the type of work required, although it does not directly address the attribution of changing risk to specific drivers. There is an urgent need for much more research on this problem. 
THE ROLE OF SCENARIOS. As discussed in the "Principles of risk assessment" section, risk assessment situations in which likelihoods cannot be quantified with precision are by no means unusual. Strategic planning in government and business routinely makes use of scenarios as tools to inform thinking about future possibilities and how to manage them. Thus, scenarios are the obvious tool to describe future climate in ways that are relevant to decision-makers. The impacts and consequences of climate scenarios can be explored in considerable quantitative detail, using metrics that range from meteorological (e.g., rainfall rate) to those that are most decision relevant (e.g., flood level, numbers of people affected, and economic loss). This characterization of impacts must, of course, include the uncertainty in these impacts.

Climate scenarios-in the sense used here-differ from climate projections. Climate projections, as used by the WGI community, purport to be a conditional prediction in which the product is some form of continuous likelihood distribution for a particular socioeconomic scenario. Climate scenarios are a discrete set of physically consistent and self-consistent storylines about the future, under a specified set of assumptions. Indeed, Shepherd et al. (2018) use the term "storylines" to describe climate scenarios of this type. They define a storyline as "a physically self-consistent unfolding of past events or of plausible future events," and have recently developed the concept in detail, explaining how it can be used to synthesize scientific evidence in decision-relevant terms.

Many national climate scenarios have been developed (e.g., www.climatescenarios.nl/, http://scenarios .globalchange.gov), but interestingly, discrete global or regional climate scenarios have not been widely used, arguably because the WGI climate science community has not promoted them. By contrast, socioeconomic scenarios have long been used by the IPCC (e.g., O'Neill et al. 2014). However, for the purposes of risk assessment, there is little difference between our knowledge/ ignorance of (say) future population growth and our knowledge/ignorance of (say) the future rate of global warming, so it would be helpful for decision-makers if the same tools-scenarios-were used to communicate this knowledge. Such an approach would be in line with King et al.'s (2015) fifth principle of risk assessment: take a holistic approach. Decision-relevant climate scenarios could usefully be developed to sample all the major dimensions of epistemic uncertainty (e.g., rapid economic growth, high greenhouse gas emissions, and high climate sensitivity).
As has already been emphasized, high-impact scenarios are of special importance for risk assessment. Sutton (2018) proposed the development of "physically plausible high impact scenarios" (PPHIS) as a specific tool for the WGI community to assess and communicate the relevant scientific evidence.

\section{CONCLUSIONS AND FURTHER IMPLICA-}

TIONS. For decision-makers, climate change is a problem in risk assessment and risk management. It is, therefore, surprising that the needs and lessons of risk assessment have not featured more prominently in the consideration of priorities for physical climate science $^{3}$ or in the WGI contributions to the major IPCC assessment reports. In this article, I have argued that this state of affairs is a result of the siloing of climate science between different disciplines (e.g., between the three IPCC working groups), but it has been exacerbated by a widespread view that the job of the WGI community is to provide predictions and projections (with a focus on likelihood rather than risk) and that risk assessment is a job for others. This view, I have argued, is incorrect. Risk assessment requires the consideration of impacts as well as likelihood. Furthermore, impacts must be assessed and quantified using a wide range of variables, and the WGI community is the appropriate group to assess impacts and risks in terms of decision-relevant physical climate variables. Future WGI reports should address this requirement.

There is also a need to recognize explicitly that, while some quantitative bounds can be assessed and potentially narrowed, it will never be possible to quantify with precision the likelihood that future climate change will take a particular form (e.g., magnitude). Consequently, an important task for the WGI community is to develop discrete sets of climate scenarios, which individually are not associated with a specific probability but collectively are designed to span the relevant uncertainty in the climate response to anthropogenic forcing (not merely the "likely range" for specific socioeconomic scenarios). The "storyline" method of Shepherd et al. (2018) offers a powerful approach. This work should include systematic attention to identifying and developing potential high-impact scenarios, even if their likelihood is

\footnotetext{
${ }^{3}$ Even the latest WCRP Strategic Plan 2019-28 (www.wcrp -climate.org/wcrp-sp) hardly mentions risk and includes no specific consideration of risk assessment needs.
} 
considered low (Sutton 2018). Impacts, including the uncertainty in impacts, should be assessed for each climate scenario.

King et al. (2015) emphasize that risks must always be assessed in relation to objectives. In the case of climate change, the relevant objectives relate to i) mitigation and ii) adaptation. For mitigation, specific priorities for WGI include the following:

1) Develop a discrete set of global climate scenarios. These should include scenarios for, for example, high climate sensitivity or high transient climate response to cumulative $\mathrm{CO}_{2}$ emissions (TCRE). The design of such scenarios should be based on understanding of the relevant Earth system processes.

2) For each global climate scenario, quantify the conditional impacts

a) on the remaining carbon budget to reach specific warming targets (e.g., IPCC 2018) and

b) on a range of decision-relevant physical climate variables (e.g., global sea level rise, major changes in regional climates such as monsoons, the likelihood of triggering irreversible melting of ice sheets).

For adaptation, specific priorities include the following:

1) Quantify current risks, in particular, the current likelihood of a wide range of decisionrelevant high impact physical events (notably extreme weather), including multihazard and correlated risks. In this area, there is an urgent need for research to address the attribution of changing risks to specific drivers.

2) To assess future risks,

a) develop regional climate scenarios (a discrete set for each chosen region). On regional scales, changes in atmospheric circulation are potentially as important as changes in global mean temperature, so regional scenarios must be designed accordingly. These should include specific high-impact scenarios, for example, associated with a shutdown in the AMOC or an abrupt shift in monsoon circulations.

b) For each regional climate scenario, quantify the conditional impacts and risks. As for current risk, this assessment should include a wide range of decision-relevant high-impact physical events, including multihazard and correlated risks.

These priorities also have consequences for climate modeling. For example, the importance of modeling strategies to quantify current risks was already highlighted in the "Some consequences" section. This is one area where new model intercomparison projects (MIPs) should be considered (e.g., a "RISK MIP," possibly based on the d4PDF experimental design). In this case, large ensembles that sample internal variability (e.g., Kay et al. 2015), at the highest resolutions possible (to capture high-impact weather), are a key requirement. A second area is the development of appropriate global and regional climate scenarios. Here, large ensembles are also required-in this case particularly to define adequately the climate response to anthropogenic forcing-but high resolution may be a lower priority. The 10-member ScenarioMIP experiments (O'Neill et al. 2016) are a step in the right direction, but it should be recognized that they rely on an unprovable assumption that the current generation of models adequately spans the full uncertainty in the climate response to anthropogenic forcing, and furthermore, these experiments involve no focused attempt to consider properly the full range of low-likelihood, high-impact scenarios. The need to assess the impacts of specific climate scenarios, including low-likelihood scenarios, is a third area. An "AMOC MIP," for example, could be used to assess the potential impacts of a significant shutdown in the AMOC.

The physical climate science WGI community cannot of course complete the task of climate change risk assessment by itself. Collaboration with other communities-notably WGII and Working Group III (WGIII) - and directly with decision-makers is essential. In the context of the IPCC Assessment Cycle, global and regional climate scenarios developed by WGI could be taken up by WGII and WGIII, and be used by national governments, to assess the full range of impacts and risks and the implications for risk management. They would also be very helpful for the production of an integrated IPCC Synthesis Report. More crosscutting IPCC reports and changes to the scoping process for the major assessment reports could also make very valuable contributions. Essential to all this, however, is for physical climate science to take its critical role in risk assessment much more seriously than hitherto. 
ACKNOWLEDGMENTS. I acknowledge funding from the U.K. Natural Environment Research Council. I thank Ted Shepherd and Ed Hawkins for many informative discussions and the three BAMS reviewers who all made very valuable suggestions for improvement.

\section{FOR FURTHER READING}

Hawkins, E., and R. Sutton, 2009: The potential to narrow uncertainty in regional climate predictions. Bull. Amer. Meteor. Soc., 90, 1095-1107, https://doi .org/10.1175/2009BAMS2607.1.

IPCC, 2012: Managing the Risks of Extreme Events and Disasters to Advance Climate Change Adaptation. C. B. Field et al., Eds., Cambridge University Press, $582 \mathrm{pp}$.

_ 2013: Summary for policymakers. Climate Change 2013: The Physical Science Basis, T. F. Stocker et al., Eds., Cambridge University Press, 1-29.

_ 2014: Summary for policymakers. Climate Change 2014: Impacts, Adaptation, and Vulnerability, C. B. Field et al., Eds., Cambridge University Press, 1-32.

_ 2018: Summary for policymakers. Global Warming of $1.5^{\circ} \mathrm{C}$, Cambridge University Press, 1-26, http:// report.ipcc.ch/sr15/pdf/sr15_spm_final.pdf.

Kay, J., and Coauthors, 2015: The Community Earth System Model (CESM) large ensemble project: A community resource for studying climate change in the presence of internal climate variability. Bull. Amer. Meteor. Soc., 96, 1333-1349, https://doi.org/10.1175 /BAMS-D-13-00255.1.

King, D., D. Schrag, Z. Dadi, Y. Qui, and A. Ghosh, 2015: Climate change: A risk assessment. Cambridge University Centre for Science and Policy Rep., 154 pp.

Mizuta, R., and Coauthors, 2017: Over 5,000 years of ensemble future climate simulations by $60-\mathrm{km}$ global and $20-\mathrm{km}$ regional atmospheric models. Bull. Amer. Meteor. Soc., 98, 1383-1398, https://doi.org/10.1175 /BAMS-D-16-0099.1.
O’Neill, B. C., E. Kriegler, K. Riahi, K. L. Ebi, S. Hallegatte, T. R. Carter, R. Mathur, D. P. van Vuuren, 2014: A new scenario framework for climate change research: the concept of shared socioeconomic pathways. Climatic Change, 122, 387-400, https://doi.org/10.1007/s10584-013-0905-2.

— , and Coauthors, 2016: The Scenario Model Intercomparison Project (ScenarioMIP) for CMIP6. Geosci. Model. Dev., 9, 3461-3482, https://doi.org/10 .5194/gmd-9-3461-2016.

Schellnhuber, H. J., 2018: Foreword. What lies beneath: The understatement of existential climate risk, Breakthrough National Centre for Climate Restoration Rep., 2-3.

Shepherd, T. G., and Coauthors, 2018: Storylines: An alternative approach to representing uncertainty in physical aspects of climate change. Climatic Change, 151, 555-571, https://doi.org/10.1007/s10584-018 $-2317-9$.

Stott, P. A., and Coauthors, 2016: Attribution of extreme weather and climate-related events. Wiley Interdiscip. Rev.: Climate Change, 7, 23-41, https://doi .org/10.1002/wcc.380.

Sutton, R. T., 2018: ESD ideas: A simple proposal to improve the contribution of IPCC WGI to the assessment and communication of climate change risks. Earth Syst. Dyn., 9, 1155-1158, https://doi .org/10.5194/esd-9-1155-2018.

Weaver, C. P., R. J. Lempert, C. Brown, J. A. Hall, D. Revell, and D. Sarewitz, 2013: Improving the contribution of climate model information to decision making: The value and demands of robust decision frameworks. Wiley Interdiscip. Rev.: Climate Change, 4, 39-60, https://doi.org/10.1002/wcc.202.

—, R. H. Moss, K. L. Ebi, P. H. Gleick, P. C. Stern, C. Tebaldi, R. S. Wilson, and J. L. Arvai, 2017: Reframing climate change assessments around risk: Recommendations for the US National Climate Assessment. Environ. Res. Lett., 12, 080201, https://doi .org/10.1088/1748-9326/aa7494. 